\title{
Immunonutrition for Adults With ARDS: Results From a Cochrane Systematic Review and Meta-Analysis
}

\author{
Ahilanandan Dushianthan, Rebecca Cusack, Victoria A Burgess, Michael PW Grocott, and \\ Philip Calder
}

\begin{abstract}
BACKGROUND: ARDS is an overwhelming systemic inflammatory process associated with significant morbidity and mortality. Several trials have evaluated the effects of pharmaconutrients, given as part of a feeding formula or as a nutritional supplement, on clinical outcomes in critical illness and ARDS. The aim of this review is to assess the effects of immunonutrition on mechanically ventilated adults with ARDS compared to the standard feeding formula. METHODS: We searched MEDLINE, EMBASE, CENTRAL, conference proceedings, and trial registries for appropriate studies up to April 2018. We performed statistical analysis according to Cochrane methodological standards. We used the GRADE approach to assess the quality of evidence for each outcome. RESULTS: We identified 10 randomized controlled trials with 1,015 participants. All of the studies compared an enteral formula or additional supplemental omega-3 fatty acids (eg, eicosapentaenoic acid, docosahexaenoic acid), $\gamma$-linolenic acid, and antioxidants. All of the studies reported mortality. For the primary outcome, there was no difference in all-cause mortality (for the longest period reported) with the use of an immunonutrition enteral formula or additional supplements of omega-3 fatty acids, $\gamma$-linolenic acid, and antioxidants (risk ratio $=0.79,95 \%$ CI $0.59-1.07$; low-quality evidence). For the secondary outcomes, we are uncertain whether immunonutrition with omega-3 fatty acids and antioxidants improves ICU length of stay, ventilator days, and oxygenation or increases harm. CONCLUSIONS: This Cochrane meta-analysis of 10 studies of varying quality examined the effects of omega-3 fatty acids and antioxidants in adults with ARDS. This intervention may produce little or no difference in all-cause mortality between groups. We are uncertain whether immunonutrition with omega-3 fatty acids and antioxidants improves ventilator days, ICU length of stay, or oxygenation due to the very low quality of evidence. Key words: acute respiratory distress syndrome; immunonutrition; omega-3 fatty acids. [Respir Care 2020;65(1):99-110. () 2020 Daedalus Enterprises]
\end{abstract}

\section{Introduction}

ARDS is characterized by refractory hypoxic respiratory failure with significant global inflammatory processes

\footnotetext{
Dr Dushianthan is affiliated with the General Intensive Care Unit, and Dr Burgess is affiliated with the Department of Anaesthetics, University Hospital Southampton NHS Foundation Trust, Southampton, UK. Dr Cusack is affiliated with the Integrative Physiology and Critical Illness Group; Dr Grocott is affiliated with the Critical Care Group, Clinical and Experimental Sciences, Faculty of Medicine; and Dr Calder is affiliated with the Human Development and Health Academic Unit, Faculty of Medicine, University of Southampton, Southampton, UK.
}

Dr Grocott has disclosed relationships with Baxter, BOC Medical (Linde Group), Eli Lilly Critical Care, Fresenius-Kabi, Smith Medical, Deltex and multi-organ dysfunction. In the lung, diffuse epithelial and endothelial injury leads to increased alveolar-capillary permeability and florid pulmonary edema. Clinically, patients present with acute refractory hypoxemia and poor

Medical, London Clinic, and Rolex. Dr Calder has disclosed relationships with Abbott Nutrition, Baxter Healthcare, Danone/Nutricia, Fresenius-Kabi, Pronova BioPharma/BASF AS, and Smartfish. The other authors have disclosed no conflicts of interest.

This article is based on a Cochrane Review published in the Cochrane Database of Systematic Reviews (CDSR) 2019, Issue 1, DOI: 10.1002/ 14651858.CD012041 (see www.thecochranelibrary.com for information). Cochrane Reviews are regularly updated as new evidence emerges and in response to feedback, and the CDSR should be consulted for the most recent version of the review. 
lung compliance, often necessitating invasive mechanical ventilation. ${ }^{1}$ Hospital mortality associated with ARDS varies between $27 \%$ and $45 \%$, depending on the severity of the disease. ${ }^{2,3}$ Survivors of ARDS have significant longterm physical, cognitive, and psychological sequelae., ${ }^{4,5}$

Immunonutrition refers to modulation of the immune system provided by specific interventions that modify dietary nutrients. ${ }^{6}$ It has long been recognized that supplementary immunonutrients may alter the course of critical illness following sepsis, trauma, and surgery. ${ }^{7}$ Several specialized enteral and parenteral formulas with immunonutrients are currently available on the market. These primarily consist of a combination of antioxidant vitamins (eg, vitamin $\mathrm{C}$, vitamin $\mathrm{E}, \beta$-carotene), trace elements (eg, selenium, zinc), essential amino acids (eg, glutamine, arginine) or essential fatty acids, such as omega-3 fatty acids (eg, eicosapentaenoic acid, docosahexaenoic acid), and $\gamma$-linolenic acid. ${ }^{8}$ ARDS is characterized by overt recruitment of neutrophils, significant release of pro-inflammatory cytokines and chemokines, and activation of procoagulant cascades and prostaglandin pathways with increased oxidative stress, causing damage to both lipids and proteins. ${ }^{9}$

In patients with ARDS, a significant imbalance in the antioxidant system with a relative increase in oxidative stress leads to increased alveolar injury. ${ }^{10-12}$ Among critically ill patients in general, supplementation of antioxidants is associated with a favorable outcome. ${ }^{13}$ Macronutrients such as glutamine and arginine also have immunomodulatory properties and have been used in several clinical trials of critically ill and surgical patients. ${ }^{14-17}$ Glutamine improves gut barrier function and can be an energy source for lymphocytes, neutrophils, and macrophages, ${ }^{18,19}$ whereas arginine deficiency, which is commonly encountered following critical illness, may impair $\mathrm{T}$ cell function. ${ }^{20}$ Omega-3 fatty acids are essential lipids, enriched in fish oil and consisting of polyunsaturated fatty acids such as eicosapentaenoic acid, $\alpha$-linolenic acid, and docosahexaenoic acid. Therapeutic supplementation of these nutrients, which have immunomodulatory properties, has been shown to moderate the inflammatory response through suppression of pro-inflammatory eicosanoid biosynthesis, ${ }^{21}$ attenuations of pulmonary neutrophil accumulation, ${ }^{22}$ reductions in lung permeability, ${ }^{23}$ and attenuation of cardiopulmonary dysfunction in animal models of lung injury. ${ }^{24}$ Furthermore, in endotoxemic rat mod-

Correspondence: Ahilanandan Dushianthan PhD, General Intensive Care Unit, University Hospital Southampton NHS Foundation Trust, Tremona Road, Southampton, UK SO16 6YD. E-mail: adushianthan@gmail.com.

DOI: $10.4187 /$ respcare. 06965 els, eicosapentaenoic acid has been shown to reduce pulmonary edema. ${ }^{25}$

Various types of immunonutrition have the potential to influence clinical outcomes in critically ill patients. ${ }^{8} \mathrm{Sev}-$ eral RCTs have investigated enteral supplementation of omega-3 fatty acids and antioxidants, which, when combined in a meta-analysis, showed a significant reduction in mortality with improvement in oxygenation for patients with acute lung injury and ARDS. ${ }^{26}$ However, recent randomized controlled trials (RCTs) and subsequent further meta-analyses have presented conflicting results, suggesting a lack of benefit and possibly even harm caused by this intervention. ${ }^{27-31}$ Among critically ill subjects, enteral supplementation of glutamine conferred no clinical benefit, ${ }^{32}$ and a large RCT showed a trend toward increased mortality associated with glutamine therapy. ${ }^{16}$ This lack of demonstrable clinical benefit in recent studies conflicts with the established literature and may be due to the heterogeneity of diseases within the population of critical care patients, or variations in the type, route, and dose of immunonutrients administered. Uncertainty arising from these conflicting results remains. This review aims to provide a comprehensive evaluation of the effects of immunonutrients for patients with ARDS, and to systematically review and critically appraise available evidence on the effects of immunonutrition compared to standard non-immunonutrition formula feeding on mechanically ventilated adults (age $\geq 18$ y) with ARDS.

\section{Methods}

This is an abridged version of a previously published Cochrane systematic review and meta-analysis. Details of the methodology are available in the Cochrane version of this systematic review; Cochrane reviews are regularly updated as new evidence emerges and in response to feedback, and the Cochrane Database of Systematic Reviews should be consulted for the most recent version of the review. ${ }^{33}$ We included all studies involving mechanically ventilated adult participants (age $\geq 18$ y) with ARDS as defined by the Berlin definition of $\mathrm{ARDS}^{2}$ or, for older studies, as defined by American-European Consensus Criteria for both ARDS and acute lung injury. ${ }^{34}$ Eligible trials included intervention groups consisting of participants given enteral or parenteral immunonutrients, additionally supplemented with or as part of a nutritional formula. In comparison, control groups included participants who received placebo or standard nutrition with a non-immunonutrient formula feed.

\section{Outcome Measures}

The primary outcome was all-cause mortality (for the longest period reported). Secondary outcomes were 28-d 
mortality, ICU length of stay (LOS) and ICU-free days at day 28, ventilator days and ventilator-free days at day 28 , hospital LOS, indices of oxygenation (measured as $\mathrm{P}_{\mathrm{aO}_{2}} / \mathrm{F}_{\mathrm{IO}_{2}}$ at day 4 and day 7), other organ failure (ie, a change in organ failure scores: Sequential Organ Failure Assessment [SOFA] score, Multiple Organ Dysfunction Score [MODS]; recorded as the number of subjects with new organ failure developed during the study period), nosocomial infection (ie, additional infection developed during the hospital stay and reported anytime during the study period), and adverse events (eg, author-defined cardiac events, gastrointestinal events, and total adverse events reported anytime during the study period).

\section{Searches, Data Extraction, and Management}

We searched on the Cochrane Central Register of Controlled Trials (CENTRAL; 2018, Issue 4, April) in the Cochrane Library), MEDLINE (OVID SP; 1966 to April 2018, week 3), and EMBASE (OVID SP; 1988 to April 2018, week 3). Two review authors (AD, RC) independently screened appropriate studies for study characteristics and outcomes. We resolved disagreements by further discussion and with the involvement of a third review author (MG). When necessary, we contacted trial authors to request additional information.

\section{Assessment of Risk of Bias in Included Studies}

We assessed the risk of bias of included studies according to criteria presented in the Cochrane Handbook for Systematic Reviews of Interventions. ${ }^{35} \mathrm{We}$ assigned the included studies to low risk of bias when all domains were satisfied, high risk of bias if one or more domains were inadequate, or unclear risk of bias, according to the criteria provided in the Cochrane risk of bias tool. We used the following 6 domains to assess the risk of bias in the included studies: selection bias, reporting bias, performance bias, detection bias, attrition bias, and any other bias.

\section{Measures of Treatment Effect}

We based the outcome analysis on intention-to-treat. We expressed dichotomous outcomes as risk ratios with 95\% CIs, and continuous variables as mean differences with standard deviations. We assessed the clinical heterogeneity of studies in relation to the study population, interventions, and outcome measures. We also assessed inconsistencies and variability in outcomes among studies using the $\mathrm{I}^{2}$ statistic. We assumed substantial statistical heterogeneity when the $\mathrm{I}^{2}$ statistic exceeded $40 \% .{ }^{35}$ We used graphical evidence of reporting biases via contourenhanced funnel plots with a subsequent Harbord or Egger test. ${ }^{36,37}$

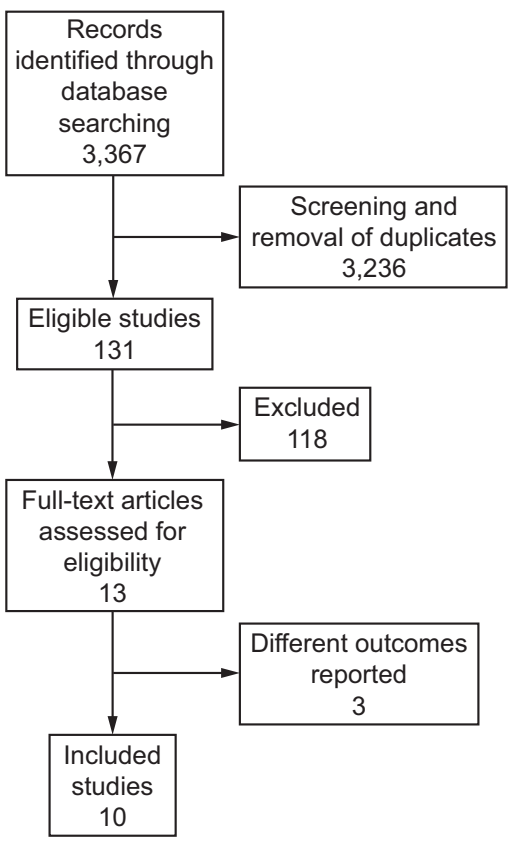

Fig. 1. Flow chart.

\section{Subgroup and Sensitivity Analyses}

We conducted subgroup analyses for the primary outcome according to type of intervention, route of intervention (parenteral/enteral), mode of intervention (continuous/bolus), and intervention duration (measured in days). We also performed a sensitivity analysis for the primary outcome while excluding studies with high risk of bias. Continuous data for the secondary outcomes were skewed. We conducted a sensitivity analysis for these secondary outcomes by $\log$ transformation. Analyses for 2 of the secondary outcomes (ie, ICU-free days and ventilator-free days at day 28) were sensitive to statistical methods; we also performed sensitivity analyses for these outcomes using both fixed-effect and random-effects models to address these issues.

\section{Quality of Evidence}

We used the principles of the GRADE system to assess the quality of the body of evidence associated with specific outcomes. ${ }^{38}$ Through the GRADE approach, we appraised the quality of a body of evidence based on the extent to which one can be confident that an estimate of effect or association reflects the item being assessed. Assessment of the quality of a body of evidence considers the within-study risk of bias (methodological quality), directness of evidence, heterogeneity of data, the precision of effect estimates, and risk of publication bias. 
Table 1. Characteristics of Included Studies

\begin{tabular}{|c|c|c|c|c|c|c|c|}
\hline Study & Country & Setting & Population & Subjects, $N$ & $\begin{array}{l}\text { Baseline } \mathrm{P}_{\mathrm{aO}_{2}} / \mathrm{F}_{\mathrm{IO}_{2}} \\
\mathrm{~mm} \mathrm{Hg}\end{array}$ & Intervention & $\begin{array}{l}\text { Duration of } \\
\text { Intervention }\end{array}$ \\
\hline Elamin $^{39}$ & USA & Multi-center & ARDS & 17 & $\begin{array}{l}\text { Control: } 138 \\
\text { Intervention: } 157\end{array}$ & $\begin{array}{l}\text { Continuous enteral EPA + DHA + } \\
\text { GLA }+ \text { antioxidants }\end{array}$ & $7 \mathrm{~d}$ \\
\hline Gadek $^{40}$ & USA & Multi-center & ARDS & 146 & $\begin{array}{l}\text { Control: } 170 \\
\text { Intervention: } 155\end{array}$ & $\begin{array}{l}\text { Continuous enteral EPA + DHA + } \\
\text { GLA + antioxidants }\end{array}$ & $\geq 4 \mathrm{~d}$ \\
\hline Grau-Camona ${ }^{46}$ & Spain & Multi-center & Septic ventilated & 160 & $\begin{array}{l}\text { Control: } 122 \\
\text { Intervention: } 130\end{array}$ & $\begin{array}{l}\text { Continuous enteral EPA + DHA + } \\
\text { GLA + antioxidants }\end{array}$ & $28 \mathrm{~d}$ \\
\hline Gupta $^{41}$ & India & Single-center & ARDS & 61 & $\begin{array}{l}\text { Control: } 199 \\
\text { Intervention: } 145\end{array}$ & Intravenous omega-3 fatty acids & $14 \mathrm{~d}$ \\
\hline Parish $^{42}$ & Iran & Multi-center & ARDS & 58 & $\begin{array}{l}\text { Control: } 145 \\
\text { Intervention: } 149\end{array}$ & Bolus omega-3 gels & $14 \mathrm{~d}$ \\
\hline Pontes-Arruda ${ }^{43}$ & Brazil & Multi-center & Septic ARDS & 165 & $\begin{array}{l}\text { Control: } 158 \\
\text { Intervention: } 156\end{array}$ & $\begin{array}{l}\text { Continuous enteral EPA + DHA + } \\
\text { GLA + antioxidants }\end{array}$ & $28 \mathrm{~d}$ \\
\hline Rice $^{27}$ & USA & Multi-center & ARDS & 272 & $\begin{array}{l}\text { Control: } 172 \\
\text { Intervention: } 160\end{array}$ & $\begin{array}{l}\text { Bolus enteral EPA + DHA + } \\
\text { GLA + antioxidants }\end{array}$ & $21 \mathrm{~d}$ \\
\hline Shirai ${ }^{44}$ & Japan & Single-center & Septic ARDS & 46 & Not presentable & $\begin{array}{l}\text { Continuous enteral EPA + DHA + } \\
\text { GLA + antioxidants }\end{array}$ & $14 \mathrm{~d}$ \\
\hline Singer ${ }^{45}$ & Israel & Single-center & ARDS & 100 & $\begin{array}{l}\text { Control: } 231 \\
\text { Intervention: } 208\end{array}$ & $\begin{array}{l}\text { Continuous enteral EPA + DHA + } \\
\text { GLA + antioxidants }\end{array}$ & $14 \mathrm{~d}$ \\
\hline Stapleton ${ }^{28}$ & USA & Multi-center & ARDS & 90 & $\begin{array}{l}\text { Control: } 172 \\
\text { Intervention: } 155\end{array}$ & $\begin{array}{l}\text { Bolus enteral fish oil (EPA + } \\
\text { DHA) }\end{array}$ & $14 \mathrm{~d}$ \\
\hline \multicolumn{8}{|c|}{$\begin{array}{l}\text { DHA }=\text { docosahexaenoic acid } \\
\text { EPA }=\text { eicosapentaenoic acid } \\
\text { GLA }=\lambda \text {-linolenic acid }\end{array}$} \\
\hline
\end{tabular}

\section{Results}

\section{Characteristics of Included Studies}

The combined search yielded 3,367 studies for possible inclusion. We retrieved a total of 13 publications reporting 10 RCTs (Fig. 1). ${ }^{27,28,39-46}$ Three studies were conducted exclusively on subjects with sepsis-induced ARDS. ${ }^{43,44,46}$ We did not identify any other intervention apart from omega-3 fatty acids (ie, eicosapentaenoic acid and docosahexaenoic acid) and $\gamma$-linolenic acid with or without antioxidant-based enteral formula or supplementation. Several clinical trials investigated the use of glutamine supplementation for critical illness, but none specifically focused on ARDS. ${ }^{32}$

Among the 10 included studies, 6 of them used a similar enteral preparation (Oxepa; Abbott Nutrition/Abbott Laboratories, Columbus, Ohio) continuously supplemented with eicosapentaenoic acid, docosahexaenoic acid, $\gamma$-linolenic acid, and antioxidants (Table 1). ${ }^{36,37,40-43}$ Four of these 6 studies used an isocaloric high-fat formulation (Pulmocare; Abbott Nutrition/Abbott Laboratories, Columbus, Ohio) for control groups. ${ }^{39,40,43,45}$ The remaining 2 studies used a carbohydrate-based control formulation (Ensure Plus or Ensure Liquid; Abbott Laboratories, East Windsor, New Jersey). ${ }^{44,46}$ Three studies used additional enteral supple- mentation of fish oil, ${ }^{28}$ omega-3 gels, ${ }^{42}$ or intravenous formulation with $10 \%$ fish oil,${ }^{41}$ and control groups received the same enteral feeding formulation as intervention groups. One study gave boluses of a high-fat formulation enriched in eicosapentaenoic acid, docosahexaenoic acid, $\gamma$-linolenic acid, and antioxidants and compared this with isocaloric/isovolemic carbohydrate-rich control nutrition. ${ }^{27}$ Five studies defined a target enteral nutrition delivery rate of $50 \%$ to $75 \%$ of basal energy expenditure/resting energy expenditure (BEE/REE) for the first $24 \mathrm{~h}$ with variable increments to achieve $70 \%$ to $100 \%$ of BEE/REE. $39,40,43-45$ The duration of intervention was $\leq 7 \mathrm{~d},{ }^{40,41} 14 \mathrm{~d}, 28,41,42,44,45$ $21 \mathrm{~d},{ }^{27}$ or $28 \mathrm{~d} .{ }^{43,46}$

\section{Risk of Bias in Included Studies}

We identified an increased risk of bias among 6 studies (Fig. 2). Authors of 4 studies reported that they were industry-supported. ${ }^{40,43,45,46} \mathrm{We}$ rated these studies as having an unclear risk of bias.

\section{Primary Outcome: All-Cause Mortality (Longest Period Reported)}

All studies reported mortality. The range of reported mortality varied between studies: six studies reported this 


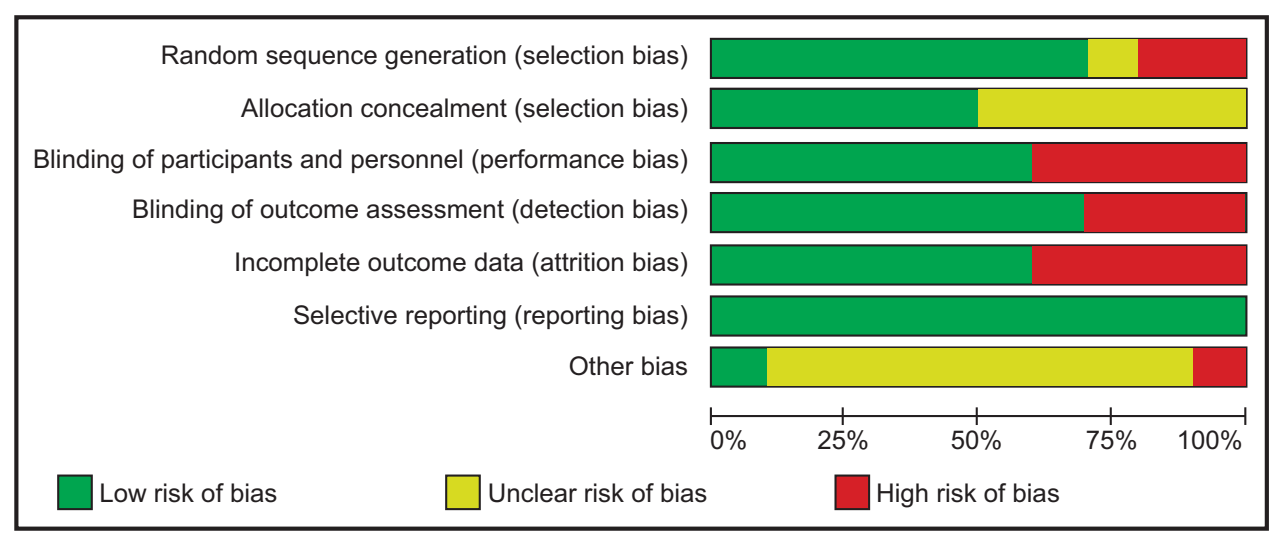

Fig. 2. Risk of bias graph: review authors' judgments about each risk of bias item presented as percentages across all included studies.

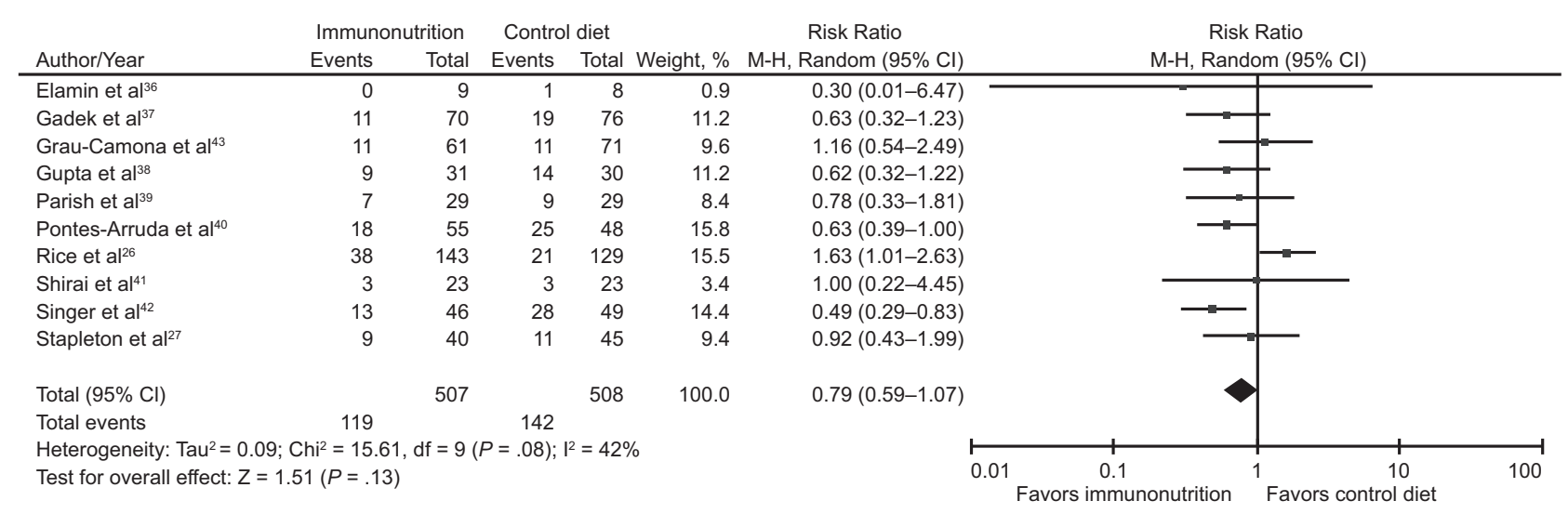

Fig. 3. Forest plot for the comparison omega-3 fatty acids and antioxidants versus placebo or standard nutrition. Outcome shown is all-cause mortality (longest period reported).

outcome at day $28,39,41-43,45,46$ three studies at day $60,27,28,44$ and one for the study duration ${ }^{40}$. There was no evidence for the use of omega-3 fatty acids and antioxidants for reducing mortality at the longest period reported (risk ratio $=0.79,95 \%$ CI $0.59-1.07 ; \mathrm{I}^{2}=42 \%$; participants $=1,015)($ Fig. 3$)$. The pooled control group mortality rate was $28 \%$, and the pooled intervention group mortality rate was $23.5 \%$ for the longest period reported. Overall mortality varied between studies and ranged from $6 \%$ to $43 \%$. A funnel plot of the primary outcome prepared to test the effect of publication bias showed no evidence of data asymmetry (Egger's regression test, with $P=.81$ ) (Fig. 4). We downgraded the quality of evidence by two levels due to inconsistency from clinical and methodological heterogeneity and indirectness for intervention and comparator.

\section{Secondary Outcomes}

Six studies with 466 participants reported 28-d mortality. ${ }^{39,41-43,45,46}$ We noted uncertain evidence for use of omega-3 fatty acids and antioxidants in terms of mortality at $28 \mathrm{~d}$ (risk ratio $=0.64,95 \% \mathrm{CI} 0.49-0.84 ; \mathrm{I}^{2}=0 \%$ )
(Fig. 5). This analysis was limited by the small number of participants, which accounted for $<50 \%$ of the total participants included.

There is uncertain evidence for the use of omega-3 fatty acids and antioxidants for the improvements in secondary outcomes of ICU LOS, duration of mechanical ventilation, ICU-free days at day 28 and ventilation-free days at day 28 (Table 2). There was also uncertain evidence for the use of omega- 3 fatty acids and antioxidants in terms of improvements in $\mathrm{P}_{\mathrm{aO}_{2}} / \mathrm{F}_{\mathrm{IO}_{2}}$ ratio at day 4 and at day 7 , reported new organ failures, nosocomial infections, and adverse events (Table 2). We downgraded the quality of evidence by 3 levels due to increased risk of bias, inconsistency due to clinical and methodological heterogeneity, and indirectness for both intervention and comparator.

\section{Subgroup Analysis}

For the primary outcome, we performed a subgroup analysis for types of control nutrition given, route of administration (parenteral/enteral), mode (continuous/ 


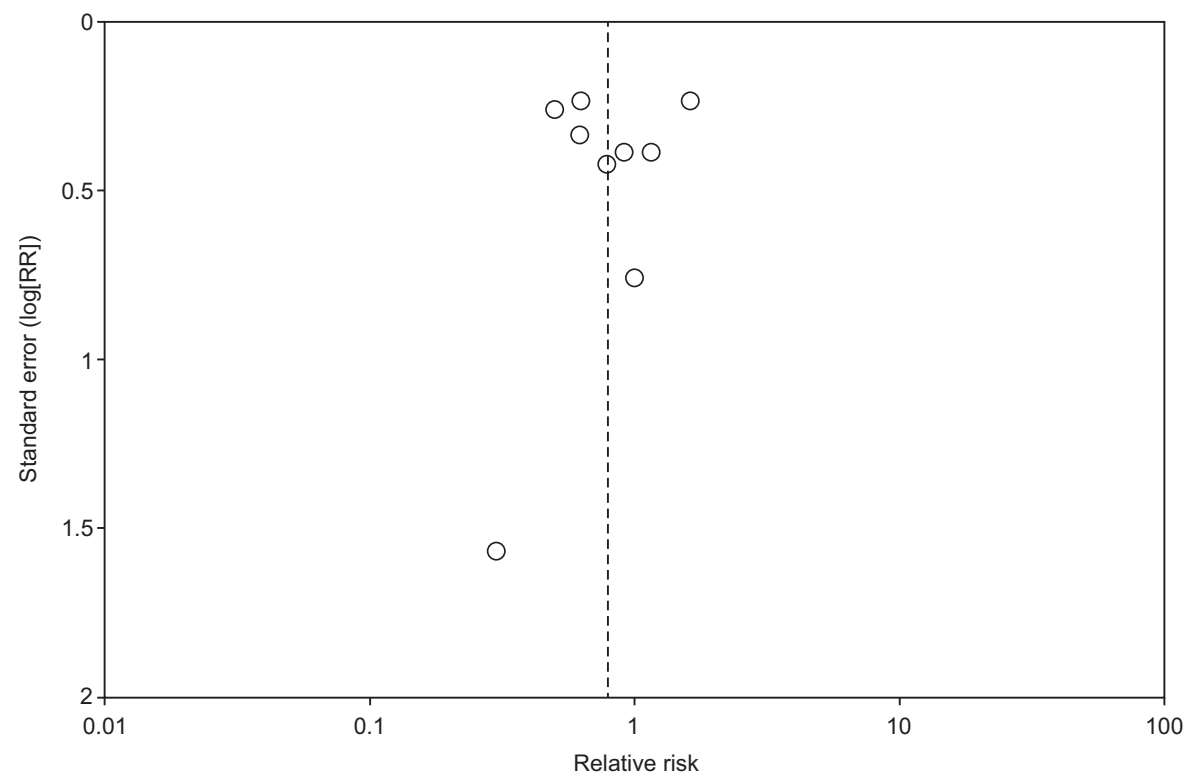

Fig. 4. Funnel plot of comparison: omega-3 fatty acids and antioxidants versus placebo or standard nutrition. Outcome shown is all-cause mortality (longest period reported). No data asymmetry with Egger's regression test $(P=.81)$. RR $=$ relative risk.

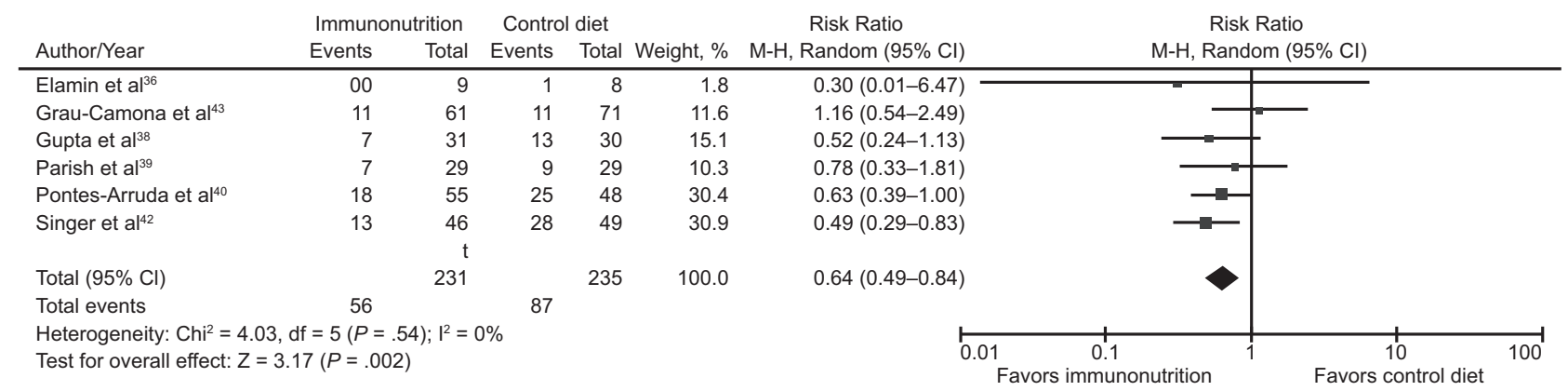

Fig. 5. Forest plot for the comparison omega-3 fatty acids and antioxidants versus placebo or standard nutrition. Outcome shown is $28-d$ mortality (longest period reported).

bolus), and the duration of intervention (Table 3). We are uncertain of any mortality benefit when investigators compared an omega-3 fatty acid or antioxidant group with a control group administered with a lipid-rich or carbohydrate-rich formula or as additional supplementation of omega-3 fatty acids. Only one study gave intravenous omega-3 fatty acid supplementation. ${ }^{41}$ All remaining studies gave enteral supplementation. We are also uncertain of any mortality benefit derived by different routes of administration (enteral or intravenous), either as continuous or bolus supplementation. We are also uncertain of any mortality benefit associated with duration of intervention for $<7 \mathrm{~d}, 14 \mathrm{~d}$, or for $28 \mathrm{~d}$ (Table 3). For all subgroup analyses, we downgraded the quality of evidence by 3 levels due to increased risk of bias, inconsistency due to clinical and methodological heterogeneity, and indirectness for intervention and comparator.

\section{Sensitivity Analysis}

We conducted a sensitivity analysis for the primary outcome while excluding studies with high risk of bias. We included 4 studies in the analysis. ${ }^{27,28,40,42} \mathrm{We}$ found no evidence for the use of omega- 3 fatty acids and antioxidants in reducing mortality at the longest period reported (Table 4). Most data for continuous secondary outcomes, such as ICU LOS, ICU-free days at day 28 , ventilator days, ventilator-free days at day 28 , and hospital LOS, were skewed. Therefore, we also performed sensitivity analysis for these outcomes from log-transformed data. Despite this transformation, we are uncertain whether this intervention confers any beneficial effect on these secondary outcomes (Table 4). We also performed sensitivity analysis using both fixed-effect and random-effects models for secondary outcomes of 
Table 2. Summary Statistical Analysis for Secondary Outcomes

\begin{tabular}{|c|c|c|c|c|}
\hline Outcome & Studies, no. & Participants, $n$ & Statistical Method & Effect Size \\
\hline ICU length of stay & 8 & 639 & MD (IV, Random, 95\% CI) & $-3.09(-5.19$ to -0.99$)$ \\
\hline ICU-free days at day 28 & 5 & 607 & MD (IV, Random, 95\% CI) & $3.44(-1.17$ to 8.06$)$ \\
\hline Ventilator days & 7 & 581 & MD (IV, Fixed, 95\% CI) & $-2.24(-3.77$ to -0.71$)$ \\
\hline Ventilator-free days at day 28 & 6 & 665 & MD (IV, Random, 95\% CI) & $2.15(-0.91$ to 5.22$)$ \\
\hline Hospital length of stay & 3 & 291 & MD (IV, Fixed, 95\% CI) & $-2.79(-7.01$ to 1.44$)$ \\
\hline $\mathrm{P}_{\mathrm{aO}_{2}} / \mathrm{F}_{\mathrm{IO}_{2}}$ ratio at day 4 & 8 & 676 & MD (IV, Random, 95\% CI) & 38.88 (10.75 to 67.02$)$ \\
\hline $\mathrm{P}_{\mathrm{aO}_{2}} / \mathrm{F}_{\mathrm{IO}_{2}}$ ratio at day 7 & 9 & 465 & MD (IV, Random, 95\% CI) & $23.44(1.73$ to 45.15$)$ \\
\hline Subjects with new organ failure & 2 & 249 & RR (M-H, Fixed, 95\% CI) & $0.45(0.32$ to 0.63$)$ \\
\hline Subjects with nosocomial infections & 3 & 450 & RR (M-H, Fixed, 95\% CI) & $1.01(0.76$ to 1.33$)$ \\
\hline \multicolumn{5}{|l|}{ Adverse events } \\
\hline Cardiac & 3 & 339 & RR (M-H, Random, 95\% CI) & $0.87(0.09$ to 8.46$)$ \\
\hline Gastrointestinal & 4 & 427 & RR (M-H, Fixed, 95\% CI) & $1.11(0.71$ to 1.75$)$ \\
\hline Total adverse events & 5 & 517 & RR (M-H, Fixed, 95\% CI) & 0.91 (0.67 to 1.23$)$ \\
\hline $\begin{array}{l}\mathrm{MD}=\text { mean difference } \\
\mathrm{IV}=\text { inverse variance } \\
\text { Random = random-effects model } \\
\text { Fixed = fixed-effects model } \\
\mathrm{RR}=\text { relative risk } \\
\mathrm{M}-\mathrm{H}=\text { Mantel-Haenszel }\end{array}$ & & & & \\
\hline
\end{tabular}

Table 3. Summary of Subgroup Analysis for Primary Outcomes

\begin{tabular}{|c|c|c|c|c|}
\hline Subgroup Analysis for Primary Outcome & Studies, no. & Participants, $n$ & Statistical Method & Effect Size \\
\hline \multicolumn{5}{|l|}{ Type of intervention } \\
\hline $\begin{array}{l}\text { Immunonutrition formula compared with lipid- } \\
\text { based control diet }\end{array}$ & 4 & 361 & RR (M-H, Random, 95\% CI) & $0.57(0.42-0.78)$ \\
\hline $\begin{array}{l}\text { Immunonutrition formula compared with } \\
\text { carbohydrate- based control diet }\end{array}$ & 2 & 178 & RR (M-H, Random, 95\% CI) & $1.13(0.57-2.22)$ \\
\hline $\begin{array}{l}\text { Immunonutrition supplemented to same } \\
\text { intervention and control diet }\end{array}$ & 3 & 204 & RR (M-H, Random, 95\% CI) & $0.75(0.48-1.15)$ \\
\hline $\begin{array}{l}\text { Immunonutrition supplemented to different } \\
\text { intervention and control diet }\end{array}$ & 1 & 272 & RR (M-H, Random, 95\% CI) & $1.63(1.01-2.63)$ \\
\hline \multicolumn{5}{|l|}{ Route of intervention } \\
\hline Enteral & 9 & 954 & RR (M-H, Random, 95\% CI) & $0.82(0.59-1.14)$ \\
\hline Intravenous & 1 & 61 & RR (M-H, Random, 95\% CI) & $0.62(0.32-1.22)$ \\
\hline \multicolumn{5}{|l|}{ Mode of intervention } \\
\hline Continuous infusion & 7 & 600 & RR (M-H, Random, 95\% CI) & $0.64(0.49-0.83)$ \\
\hline Bolus supplementation & 3 & 415 & RR (M-H, Random, 95\% CI) & $1.17(0.72-1.88)$ \\
\hline \multicolumn{5}{|l|}{ Duration of intervention } \\
\hline Duration $<7 \mathrm{~d}$ & 2 & 163 & RR (M-H, Random, 95\% CI) & $0.61(0.32-1.17)$ \\
\hline Duration $14 \mathrm{~d}$ & 5 & 345 & RR (M-H, Random, 95\% CI) & $0.64(0.47-0.89)$ \\
\hline Duration $21 \mathrm{~d}$ & 1 & 272 & RR (M-H, Random, 95\% CI) & $1.63(1.01-2.63)$ \\
\hline Duration $28 \mathrm{~d}$ & 2 & 235 & RR (M-H, Random, 95\% CI) & $0.79(0.44-1.44)$ \\
\hline \multicolumn{5}{|l|}{$\begin{array}{l}\mathrm{RR}=\text { relative risk } \\
\mathrm{M}-\mathrm{H}=\text { Mantel-Haenszel } \\
\text { Random = random-effects model }\end{array}$} \\
\hline
\end{tabular}

ICU-free days and ventilator-free days at day 2828 . Results were sensitive to the type of analytical method used (ie, random-effects model or fixed-effect model) (Table 5).

\section{Discussion}

We identified 10 RCTs evaluating effects of omega-3 fatty acids, eicosapentaenoic acid, docosahexaenoic acid, 
Table 4. Sensitivity Analysis for Primary Outcomes*

\begin{tabular}{|c|c|c|c|c|}
\hline Outcomes & Studies, no. & Participants, $n$ & Statistical Method & Effect Size \\
\hline $\begin{array}{l}\text { All-cause mortality (excluding studies with } \\
\text { high risk of bias) }\end{array}$ & 4 & 561 & RR (M-H, Random, 95\% CI) & $0.98(0.60-1.58)$ \\
\hline ICU length of stay, log days & 8 & 639 & MD (IV, Fixed, 95\% CI) & $0.08(-0.23$ to 0.06$)$ \\
\hline ICU-free days at day $28, \log$ days & 5 & 607 & MD (IV, Random, 95\% CI) & $0.34(0.00-0.68)$ \\
\hline Ventilator days, log days & 7 & 581 & MD (IV, Fixed, 95\% CI) & $-0.08(-0.24$ to 0.07$)$ \\
\hline Ventilator-free days at day $28, \log$ days & 6 & 665 & MD (IV, Fixed, 95\% CI) & $0.06(0.06-0.18)$ \\
\hline Hospital length of stay, log days & 3 & 291 & MD (IV, Fixed, 95\% CI) & $-0.05(-0.34$ to 0.24$)$ \\
\hline $\begin{array}{l}\text { * Excluding studies with high risk of bias and for the seco } \\
\mathrm{RR}=\text { relative risk } \\
\mathrm{M}-\mathrm{H}=\text { Mantel-Haenszel } \\
\mathrm{MD}=\text { mean difference } \\
\mathrm{IV}=\text { inverse variance } \\
\text { Random = random-effects model } \\
\text { Fixed = fixed-effects model }\end{array}$ & comes with skewec & & & \\
\hline
\end{tabular}

Table 5. Sensitivity Analysis for ICU-Free Days and Ventilator-Free Days at Day 28

\begin{tabular}{llcl}
\hline \hline \multicolumn{1}{c}{ Outcome } & \multicolumn{1}{c}{ Statistical Analysis } & Mean Difference (95\% CI) \\
\hline ICU-free days at day 28 & MD (IV, Random, 95\%) & $3.44(-1.17$ to 8.06) & $1.95(0.42-3.48)$ \\
ICU-free days at day 28 & MD (IV, Fixed, 95\%) & $2.15(-0.91$ to 5.22) \\
Ventilator-free days at day 28 & MD (IV, Random, 95\%) & $1.00(0.06-1.94)$ & .01 \\
Ventilator-free days at day 28 & MD (IV, Fixed, 95\%) & & \\
Sensitivity analysis was performed using a fixed-effects model and a random-effects model. & & \\
MD = mean difference & & \\
IV = inverse variance & & \\
\hline
\end{tabular}

$\gamma$-linolenic acid, and antioxidants, given as a supplement or as part of an enteral nutrition formula. We identified no clinical trials with any other specific immunonutrition intervention for this patient population. We found no evidence that this type of nutrition improves the primary outcome of all-cause mortality at the longest period reported. Included studies showed clinical heterogeneity with respect to type, mode, and duration of intervention provided, as well as the type of enteral nutrition formulation received by the control group. We performed subgroup analysis according to different interventions in the control group. Although we noted a statistical reduction in mortality when omega- 3 fatty acids and antioxidants were compared with a lipid-rich enteral formula, we are uncertain due to very low-quality evidence whether this intervention improves mortality in the ARDS population. We also found uncertain evidence regarding reductions in duration of mechanical ventilation, ICU LOS, improvement in oxygenation, and increased adverse events with this intervention.

We performed appropriate and thorough searches of electronic databases to identify suitable studies. We applied no restrictions. We obtained additional study details from study authors when possible. Our meta-analysis incorporated 10 clinical trials of 1,015 participants investigating immune-modifying nutrition in subjects with ARDS. All stud- ies used omega-3 fatty acid-based nutritional formula with or without antioxidants for the intervention. However, this approach was subject to significant clinical and statistical heterogeneity. Overall, pooled data did not support giving omega-3 fatty acids in combination with $\gamma$-linolenic acid and antioxidants to improve mortality in ARDS. Although results from some subgroup analyses indicate that the mortality risk ratio was reduced when the intervention was given as a continuous enteral infusion against a lipid-rich control formulation, the quality of evidence is very low. Also, the isocaloric high-fat formula used in the control group diet was enriched in omega- 6 fatty acids with a high content of linolenic acid, which may have been harmful; in other words, the beneficial effect reported by those studies may have been due to potentially harmful effects of the lipid-rich diet given to the control group.

Given that mechanical ventilator days and ICU LOS may be influenced by the death rate, more recent critical care clinical trials have widely reported ventilator-free and ICU-free days as better outcome measures. In general, ventilator-free days and ICU-free days at day 28 are used as a surrogate for overall ICU outcomes combining mortality with duration of mechanical ventilation and ICU LOS, respectively. Study results show a difference in the pooled statistical analysis between outcomes of ICU LOS 
and ICU-free days at day 28. This was also true for ventilator days and ventilator-free days at day 28. Reporting of either the number of days with ventilation or in the ICU or numbers days without ventilation or not in the ICU can be subject to bias. For this reason, we meta-analyzed both ways of reporting all available data from published trials (ventilator days/ventilator-free days at day 28 and ICU LOS/ICU-free days at day 28). Alternatively, this disagreement between our secondary composite outcomes (ICU LOS/ventilator days and ICU-free days/ventilator-free days at day 28) and individual end points could be due to inclusion and exclusion of different studies from outcome analyses, based on available outcome measures, or due to lack of standardized reporting. Nevertheless, due to this low-quality evidence, we are uncertain whether the use of an omega-3-based immune-modulating diet in ARDS improves ventilator days or ICU LOS.

Some studies did not provide adequate descriptive evidence for methods of randomization and allocation concealment. One study was unblinded, and 2 studies did not adequately clarify the blinding of outcome assessment. Significant dropouts occurred in several studies for a variety of reasons, including protocol violations and intolerance of the intervention or trial. We graded these studies as having a high risk of bias. Most studies reported anticipated outcomes and all reported mortality, although they were not adequately powered to detect differences between groups. We graded the quality of evidence for the primary outcome as low and for all secondary outcomes as very low because, despite the possibility of increased risk of bias, the primary outcome analysis included $>1,000$ subjects with $>250$ events and was not influenced by inclusion of studies with high risk of bias, as evidenced by the sensitivity analysis omitting these studies. Analyses of continuous outcome data for most secondary outcomes yielded skewed results. We were not able to obtain the raw transformed data from study authors; therefore, we performed sensitivity analysis using logarithmic transformations for these secondary outcomes. We encountered substantial statistical heterogeneity for several secondary outcomes. Significant clinical and statistical heterogeneity led to a priori defined subgroup analyses of the primary outcomes, according to the type of intervention provided.

Most included studies in this meta-analysis presented indirectness, where there were significant differences between the intervention dietary composition and control formulations. Four RCTs used a comparator enteral formula enriched in omega- 6 fatty acids. ${ }^{39,40,43,45}$ Because omega- 6 fatty acids can potentiate inflammation, this may have caused increased harm in the control group. Furthermore, one study gave bolus feeding and reported significant differences between the amount of protein given per day to the control group ( $20 \mathrm{~g}$ ) and the intervention group ( $4 \mathrm{~g}) .{ }^{27}$ There were also differences in the patient popula- tion between included studies, in that 3 studies exclusively consisted of subjects with sepsis-related ARDS. ${ }^{43,44,46}$ Etiological heterogeneity of ARDS origin may have different pathophysiological consequence, and modulation of inflammation by immunonutrition in sepsis and septic shock has produced conflicting results. ${ }^{47,48}$ Consequently, combining these studies of both pulmonary and extrapulmonary origin of subjects with ARDS may have introduced clinical heterogeneity and thus bias. Overall, we rated the quality of evidence as low to very low because of increased risk of bias, skewed data, inconsistency, and indirectness. Further research on this topic is essential both to address the overall objective of this review and to focus on specific questions. Future RCTs should consider standardized reporting of ICU outcomes to facilitate the combination and comparison of data between studies.

To our knowledge, we have identified and included all published studies on this topic. Lack of standardization in reporting outcomes resulted in some studies reporting duration of ICU stay as ICU LOS and others as ICU-free days at day 28 . We encountered similar issues when dealing with duration of mechanical ventilation. This resulted in pooling of these studies separately, which was not planned when the protocol was drafted. Lack of standardized statistical data from the included studies led to assumptions of normal distribution and calculations of standard deviation from standard error, interquartile ratio, and $P$ value, which may have introduced additional bias.

Pontes-Arruda et al ${ }^{26}$ conducted the first meta-analysis on this topic in 2008. This review included 3 earlier studies $40,43,35$ and reported a survival advantage, improvement in oxygenation (at day 4 and at day 7), and other clinical variables such as ICU-free days and ventilator-free days at day 28 with immune-enhancing diets. ${ }^{26}$ All studies included in this review gave a high-fat, low-carbohydrate formula enriched with eicosapentaenoic acid, $\gamma$-linolenic acid, and antioxidants. However, the beneficial effects seen in these trials may have been confounded by increased mortality in the control groups, possibly caused by the use of enteral formula enriched in omega- 6 fatty acids. Another meta-analysis, which only included these 3 earlier studies and focused on mortality and oxygenation, yielded the same conclusions. ${ }^{29}$

Results from a subsequent meta-analysis of 7 studies contradicted these previous positive findings and revealed that enteral supplementation of omega-3 fatty acids, $\gamma$-linolenic acid, and antioxidants provide no benefit in reducing clinical outcomes such as 28-d mortality, ventilatorfree days, and ICU-free days. ${ }^{30}$ However, these results showed an improvement in oxygenation at day 4 and at day 7 with immune-modulating diets. In comparison to the previous meta-analyses, this review included 4 additional studies, and 3 of these were methodologically different from the older studies. ${ }^{27,28,46}$ The largest of these trials (ie, 
OMEGA Trial) demonstrated no mortality benefit and was terminated early due to futility after an interim analysis. In contrast to the older studies, the intervention in this trial consisted of twice-daily bolus supplementation of omega-3 fatty acids, $\gamma$-linolenic acid, and antioxidants or the control supplement composed of an isocaloric, highcarbohydrate, low-fat feed. ${ }^{27}$ Another study supplemented enteral fish oil, ${ }^{28}$ whereas another gave a lipid-based diet with omega-3 fatty acids, $\gamma$-linolenic acid, and antioxidants as the intervention and used a high-carbohydrate, lipid-poor control enteral feed. ${ }^{46} \mathrm{~A}$ more recent meta-analysis of $6 \mathrm{RCTs}^{31}$ assessed the effect of enteral omega-3 fatty acids, $\gamma$-linolenic acid, and antioxidants. They demonstrated significant heterogeneity across studies and found no difference in clinical outcomes such as all-cause mortality, ventilator-free days, or ICU-free days. ${ }^{31}$ In this review, the authors also analyzed studies with high overall mortality and demonstrated a positive outcome with the intervention for patient groups with higher mortality, inferring potential benefit for those with severe ARDS.

Our meta-analysis was consistent with the conclusions of recently published reviews in finding no mortality benefit derived from immunomodulatory diets based on the inclusion of omega- 3 fatty acids, $\gamma$-linolenic acid, and antioxidants. We noted no improvement in ventilator-free days or ICU-free days at day 28 , and this was sensitive to analytical methods. These findings were consistent with those reported by a previous meta-analysis..$^{30}$ Our findings are also consistent with guidelines of the Society of Critical Care Medicine and the American Society for Parenteral and Enteral Nutrition for the provision of nutritional support for adult critically ill patients, which do not recommend the use of omega-3 fatty acids in the ARDS population. ${ }^{49}$

Our systematic review and meta-analysis showed no mortality benefit associated with use of immunonutrition in ARDS populations. Current data do not justify a large RCT on this topic but would support targeted proof-ofconcept studies in groups of subjects to refine the intervention. Consistent reporting of outcome measures by researchers will be important to allow combinations of results in subsequent meta-analyses. Mortality is unlikely to be the best outcome measure for such studies. Cost-effectiveness data are notably absent from current studies and should be collected in the future. The most promising areas for future evaluation include continuous supplementation with a balanced formula for both control and intervention groups with additional supplementation for the intervention group.

\section{Conclusions}

This meta-analysis evaluated 10 heterogeneous studies of varying quality and analyzed effects of omega-3 fatty acids and antioxidants in critically ill adults with ARDS. We did not find clinical trials of any other immunonutri- tion intervention provided to this patient group. Despite the inclusion of all studies in our meta-analysis, we were not able to pool all studies for every anticipated clinical outcome due to the lack of standardized outcome reporting. This may have introduced bias into our analysis. Our results suggest that no mortality benefit is derived from the use of omega-3 fatty acids or antioxidants in ARDS. Uncertain evidence suggests reductions in duration of mechanical ventilation and ICU LOS, along with improved oxygenation. The quality of evidence was very low due to several factors, including poor-quality small trials with high risk of bias, clinical and methodological heterogeneity, and issues due to imprecision and inconsistency between trials, with additional indirectness due to an imbalance in nutrition provided to the comparator groups.

\section{ACKNOWLEDGMENTS}

We thank Karen Hovhannisyan for her help in designing the searches, and Jane Cracknell, Managing Editor of the Cochrane Anaesthesia Critical and Emergency Care Group, for her assistance. We thank Bronagh Blackwood (content editor), Nathan Pace (statistical editor), Davoud Vahabzadeh and Thomas Bongers (peer reviewers), Patricia Tong (consumer referee), and Harald Herkner (coordinating editor) for their help and advice provided during the preparation of this systematic review. We also thank Rodrigo Cavallazzi (content editor), Jing Xie (statistical editor), and Todd Rice and Davoud Vahabzadeh (peer reviewers) for their help and editorial advice provided during preparation of the protocol for this systematic review.

\section{REFERENCES}

1. Dushianthan A, Grocott MP, Postle AD, Cusack R. Acute respiratory distress syndrome and acute lung injury. Postgrad Med J 2011; 87(1031):612-622.

2. ARDS Definition Task Force, Ranieri VM, Rubenfeld GD, Thompson BT, Ferguson ND, Caldwell E, et al. Acute respiratory distress syndrome: the Berlin Definition. JAMA 2012;307(23):2526-2533.

3. Bellani G, Laffey JG, Pham T, Fan E, Brochard L, Esteban A, et al. Epidemiology, patterns of care, and mortality for patients with acute respiratory distress syndrome in intensive care units in 50 countries. JAMA 2016;315(8):788-800.

4. Herridge MS, Tansey CM, Matte A, Tomlinson G, Diaz-Granados N, Cooper A, et al. Functional disability 5 years after acute respiratory distress syndrome. N Eng1 J Med 2011;364(14):1293-1304.

5. Wang CY, Calfee CS, Paul DW, Janz DR, May AK, Zhuo H, et al. One-year mortality and predictors of death among hospital survivors of acute respiratory distress syndrome. Intensive Care Med 2014; 40(3):388-396.

6. Calder PC. Immunonutrition BMJ 2003;327(7407):117-118.

7. Beale RJ, Bryg DJ, Bihari DJ. Immunonutrition in the critically ill: a systematic review of clinical outcome. Crit Care Med 1999;27(12): 2799-2805.

8. Mizock BA. Immunonutrition and critical illness: an update. Nutrition 2010;26(7-8):701-707.

9. Matthay MA, Zemans RL. The acute respiratory distress syndrome: pathogenesis and treatment. Annu Rev Pathol 2011;6:147-163.

10. Lang JD, McArdle PJ, O'Reilly PJ, Matalon S. Oxidant-antioxidant balance in acute lung injury. Chest 2002;122(6 Suppl):314S-20S.

11. Metnitz PG, Bartens C, Fischer M, Fridrich P, Steltzer H, Druml W. Antioxidant status in patients with acute respiratory distress syndrome. Intensive Care Med 1999;25(2):180-185. 
12. Schmidt R, Luboeinski T, Markart P, Ruppert C, Daum C, Grimminger $\mathrm{F}$, et al. Alveolar antioxidant status in patients with acute respiratory distress syndrome. Eur Respir J 2004;24(6):994999.

13. Heyland DK, Dhaliwal R, Suchner U, Berger MM. Antioxidant nutrients: a systematic review of trace elements and vitamins in the critically ill patient. Intensive Care Med 2005;31(3):327-337.

14. Andrews PJ, Avenell A, Noble DW, Campbell MK, Croal BL, Simpson WG, et al. Randomised trial of glutamine, selenium, or both, to supplement parenteral nutrition for critically ill patients. BMJ 2011; 342:d1542.

15. Heyland DK, Novak F, Drover JW, Jain M, Su X, Suchner U. Should immunonutrition become routine in critically ill patients? A systematic review of the evidence. JAMA 2001;286(8):944-953.

16. Heyland DK, Dhaliwal R. Role of glutamine supplementation in critical illness given the results of the REDOXS study. JPEN J Parenter Enteral Nutr 2013;37(4):442-443.

17. Novak F, Heyland DK, Avenell A, Drover JW, Su X. Glutamine supplementation in serious illness: a systematic review of the evidence. Crit Care Med 2002;30(9):2022-2029.

18. Newsholme EA, Crabtree B, Ardawi MS. Glutamine metabolism in lymphocytes: its biochemical, physiological and clinical importance. Q J Exp Physiol 1985;70(4):473-489.

19. Soares AD, Costa KA, Wanner SP, Santos RG, Fernandes SO, Martins FS, et al. Dietary glutamine prevents the loss of intestinal barrier function and attenuates the increase in core body temperature induced by acute heat exposure. Br J Nutr 2014;112(10): 1601-1610.

20. Popovic PJ, Zeh HJ 3rd, Ochoa JB. Arginine and immunity. J Nutr 2007;137(6 Suppl 2):1681S-1686S.

21. Calder PC. Immunonutrition in surgical and critically ill patients. Br J Nutr 2007;98(Suppl 1):S133-S139.

22. Mancuso P, Whelan J, DeMichele SJ, Snider CC, Guszcza JA, Karlstad MD. Dietary fish oil and fish and borage oil suppress intrapulmonary proinflammatory eicosanoid biosynthesis and attenuate pulmonary neutrophil accumulation in endotoxic rats. Crit Care Med 1997;25(7):1198-1206.

23. Mancuso P, Whelan J, DeMichele SJ, Snider CC, Guszcza JA, Claycombe KJ, et al. Effects of eicosapentaenoic and gammalinolenic acid on lung permeability and alveolar macrophage eicosanoid synthesis in endotoxic rats. Crit Care Med 1997;25(3): 523-532.

24. Murray MJ, Kumar M, Gregory TJ, Banks PL, Tazelaar HD, DeMichele SJ. Select dietary fatty acids attenuate cardiopulmonary dysfunction during acute lung injury in pigs. Am J Physiol 1995;269(6 Pt 2):H2090-H2099.

25. Sane S, Baba M, Kusano C, Shirao K, Andoh T, Kamada T, et al. Eicosapentaenoic acid reduces pulmonary edema in endotoxemic rats. J Surg Res 2000;93(1):21-27.

26. Pontes-Arruda A, Demichele S, Seth A, Singer P. The use of an inflammation-modulating diet in patients with acute lung injury or acute respiratory distress syndrome: a meta-analysis of outcome data. JPEN J Parenter Enteral Nutr 2008;32(6):596-605.

27. Rice TW, Wheeler AP, Thompson BT, deBoisblanc BP, Steingrub J, Rock P, et al. Enteral omega-3 fatty acid, gamma-linolenic acid, and antioxidant supplementation in acute lung injury. JAMA 2011; 306(14):1574-1581.

28. Stapleton RD, Martin TR, Weiss NS, Crowley JJ, Gundel SJ, Nathens $\mathrm{AB}$, et al. A phase II randomized placebo-controlled trial of omega-3 fatty acids for the treatment of acute lung injury. Crit Care Med 2011;39(7):1655-1662.

29. Dee BM, Bruno JJ, Lal LS, Canada TW. Effects of immune-enhancing enteral nutrition on mortality and oxygenation in acute lung injury and acute respiratory distress syndrome: a meta-analysis. Hospital Pharmacy 2011;46(1):33-40.

30. Zhu D, Zhang Y, Li S, Gan L, Feng H, Nie W. Enteral omega-3 fatty acid supplementation in adult patients with acute respiratory distress syndrome: a systematic review of randomized controlled trials with meta-analysis and trial sequential analysis. Intensive Care Med 2014; 40(4):504-512.

31. Li C, Bo L, Liu W, Lu X, Jin F. Enteral immunomodulatory diet (omega-3 fatty acid, gamma-linolenic acid and antioxidant supplementation) for acute lung injury and acute respiratory distress syndrome: an updated systematic review and meta-analysis. Nutrients 2015;7(7):5572-5585.

32. van Zanten AR, Dhaliwal R, Garrel D, Heyland DK. Enteral glutamine supplementation in critically ill patients: a systematic review and meta-analysis. Crit Care 2015;19:294.

33. Dushianthan A, Cusack R, Burgess VA, Grocott MPW, Calder PC. Immunonutrition for acute respiratory distress syndrome (ARDS) in adults. Cochrane Database Syst Rev 2019;1:CD012041.

34. Bernard GR, Artigas A, Brigham KL, Carlet J, Falke K, Hudson L, et al. The American-European Consensus Conference on ARDS. Definitions, mechanisms, relevant outcomes, and clinical trial coordination. Am J Respir Crit Care Med 1994;149(3 Pt 1):818-824.

35. Higgins JP, Green S. Cochrane Handbook of Systematic Reviews of Intervention Version 5.1.0 (updated in Mar 2011). The Cochrane Collaboration. 2011. Available at: https://training.cochrane.org/ handbook. Accessed August 6, 2019.

36. Egger M, Davey Smith G, Schneider M, Minder C. Bias in metaanalysis detected by a simple, graphical test. BMJ 1997;315(7109): 629-634.

37. Harbord RM, Egger M, Sterne JA. A modified test for small-study effects in meta-analyses of controlled trials with binary endpoints. Stat Med 2006;25(20):3443-3457.

38. Guyatt GH, Oxman AD, Kunz R, Vist GE, Falck-Ytter Y, Schunemann HJ, et al. What is "quality of evidence" and why is it important to clinicians? BMJ 2008;336(7651):995-998.

39. Elamin EM, Miller AC, Ziad S. Immune enteral nutrition can improve outcomes in medical-surgical patients with ards: a prospective randomized controlled trial. J Nutr Disord Ther 2012;2:109.

40. Gadek JE, DeMichele SJ, Karlstad MD, Pacht ER, Donahoe M, Albertson TE, et al. Effect of enteral feeding with eicosapentaenoic acid, gamma-linolenic acid, and antioxidants in patients with acute respiratory distress syndrome. Enteral Nutrition in ARDS Study Group. Crit Care Med 1999;27(8):1409-1420.

41. Gupta A, Govil D, Bhatnagar S, Gupta S, Goyal J, Patel S, et al. Efficacy and safety of parenteral omega 3 fatty acids in ventilated patients with acute lung injury. Indian J Crit Care Med 2011;15(2):108113.

42. Parish M, Valiyi F, Hamishehkar H, Sanaie S, Asghari Jafarabadi M, Golzari SE, et al. The effect of omega- 3 fatty acids on ARDS: a randomized double-blind study. Adv Pharm Bull 2014;4(Suppl 2):555-561.

43. Pontes-Arruda A, Aragao AM, Albuquerque JD. Effects of enteral feeding with eicosapentaenoic acid, gamma-linolenic acid, and antioxidants in mechanically ventilated patients with severe sepsis and septic shock. Crit Care Med 2006;34(9):2325-2333.

44. Shirai K, Yoshida S, Matsumaru N, Toyoda I, Ogura S. Effect of enteral diet enriched with eicosapentaenoic acid, gamma-linolenic acid, and antioxidants in patients with sepsis-induced acute respiratory distress syndrome. J Intensive Care 2015;3(1):24.

45. Singer P, Theilla M, Fisher H, Gibstein L, Grozovski E, Cohen J. Benefit of an enteral diet enriched with eicosapentaenoic acid and gamma-linolenic acid in ventilated patients with acute lung injury. Crit Care Med 2006;34(4):1033-1038.

46. Grau-Carmona T, Moran-Garcia V, Garcia-de-Lorenzo A, Heras-dela-Calle G, Quesada-Bellver B, Lopez-Martinez J, et al. Effect of an 


\section{IMMUNONUTRITION IN ADULTS WiTH ARDS}

enteral diet enriched with eicosapentaenoic acid, gamma-linolenic acid and anti-oxidants on the outcome of mechanically ventilated, critically ill, septic patients. Clin Nutr 2011;30(5):578-584.

47. Morisawa K, Fujitani S, Taira Y, Kushimoto S, Kitazawa Y, Okuchi $\mathrm{K}$, et al. Difference in pulmonary permeability between indirect and direct acute respiratory distress syndrome assessed by the transpulmonary thermodilution technique: a prospective, observational, multiinstitutional study. J Intensive Care 2014;2(1):24.
48. Fremont RD, Rice TW. Pros and cons of feeding the septic intensive care unit patient. Nutr Clin Pract 2015;30(3):344-350.

49. McClave SA, Taylor BE, Martindale RG, Warren MM, Johnson DR, Braunschweig C, et al. Guidelines for the provision and assessment of nutrition support therapy in the adult critically ill patient: Society of Critical Care Medicine (SCCM) and American Society for Parenteral and Enteral Nutrition (ASPEN). JPEN J Parenter Enteral Nutr 2016;40(2):159-211. 